\title{
The Effect of Seaweed Fertilizer 'SUNRED' on the Coloring of 'Red Globe' Grape
}

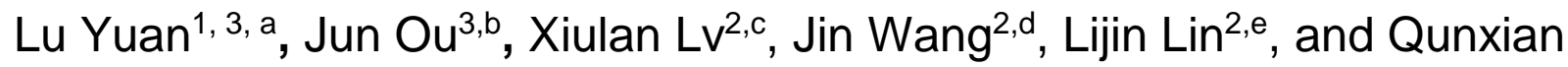 \\ Deng ${ }^{1, f *}$ \\ ${ }^{1}$ College of Horticulture, Sichuan Agricultural University, Chengdu, Sichuan, China \\ ${ }^{2}$ Institute of Pomology and Olericulture, Sichuan Agricultural University, Chengdu, China \\ ${ }^{3}$ Chengdu Agricultural College, Chengdu, Sichuan, China \\ a1054069372@qq.com, b78873116@qq.com, cxllvjj@163.com,d251040278@qq.com,

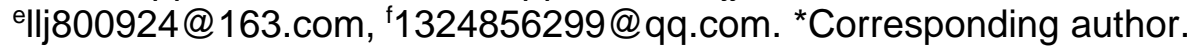

\section{Key words: SUNRED; Red Globe; coloring; anthocyanin}

Abstract: The experimental material is 'Red Globe' grape that is taking shelter from rain. Use different concentrations of SUNRED on the grapes, when the color of grape is turning. Then study the effect of color and quality of fruit. The results show that, SUNRED1000 times could promote the grape coloring. The total chlorophyll content of SUNRED 1000 treated peel was the lowest, $4.8 \mathrm{mg} / \mathrm{g}$, and the anthocyanin content was the highest, $1785 \mathrm{nmol} / \mathrm{g}$. It improved the color index of grape fruit .

\section{Introduction}

Grapes have high nutritional value and delicious taste, so many people like to buy grapes. The color and quality of fruit is an important factor in determining the value of fresh grape products. Because of the different pigment accumulated in the peel, grapes show different colors ${ }^{[1]}$. In recent years, rain shelter cultivation in grapes is maturing and developing rapidly, and becomes the new forces in fruits production. The cultivation area of table grapes is greatly increased, and the yield is also higher and higher. Especially Eurasian grapes, because of its advantages of storage and transportation easily, high yield, high benefit and so on, are highly appreciated by planters. Because the rain and heat is at the same time in the south, the light time is short, hot and humidity, and diurnal temperature difference is small, the quality of grapes is not high. It can't meet people's consumption needs of high quality and high grade fruit. Therefore, in recent years, southern China has adopted rain shelter cultivation. Grape shelter cultivation is a kind of protected cultivation, that can effectively reduce the damage caused by rain splashes in grape leaves, thereby reducing the use of pesticides and improving fruit quality ${ }^{[2]}$. However, due to the filming of the scaffold, the light is weakened, resulting in long shoots, internode growth, leaf thinning, pale leaf color, poor fruit coloring, and delayed ripening ${ }^{[3]}$.

SUNRED is a brown algae extract developed by Biolchim s.r.l in Italy to increase plant coloration and sugar accumulation. Its main components are methionine, phenylalanine, plant extracts, monosaccharides, potassium, and chelated trace elements, and has the effects of improving the coloration of fruits, increasing the soluble sugar content of fruits, promoting the ripening of fruits, and improving the storage resistance of fruits. Studies have found that seaweed fertilizer can increase the leaf area of grapes and increase the photosynthetic efficiency of the leaves ${ }^{[4]}$. In the study of seaweed fertilizer on melon, it was found that the application of seaweed fertilizer can not only improve the yield and fruit quality of melon, but also make the color of melon appearance deepen and the coloring is more even ${ }^{[5]}$.

This experiment used the Eurasian grape 'Red Globe' planted in the southern region as a test material. 'Red Globe' grapes were treated with different concentrations of SUNRED to investigate its effect on the coloration of 'Red Globe' grapes.By improving the value of goods, grape' s social and economic benefits can be increased. 


\section{Materials and Methods}

Materials and sampling. The experimental park is located in the Grape Demonstration Park in Pengshan County, Meishan City, of Sichuan Province. It is 600 meters above sea level, subtropical humid climate, mild climate, abundant rainfall, and four distinct seasons. The experimental trees are 'Red Globe' grape (medium and late ripening variety) cultivated for 7 years, that are taking shelter from rain. The planting spacing is $2 \mathrm{~m} \times 3 \mathrm{~m}$, and the tree shaped is $\mathrm{Y}$. And the experimental trees have strong tree vigor and the general cultivation and management are basically the same. At the beginning of coloring, select representative and uniform-sized clusters to hung up the tag, and sprayed SUNRED firstly. The different treat were water, SUNRED 600 times, SUNRED 800 times, SUNRED 1000 times. After 10 days, sprayed SUNRED secondly using the same spraying method at the first time. And before spraying at that day, began to sample. One sample every five days until fruit ripening. Each time, picked 20 even-sized grapes from Top, middle, and bottom of the tree. At young fruit stage, picked 120 grapes. After fruit expanding, picked 90 grapes. Brong samples back to the lab with an ice box and peeled the peel and flesh for storage in a refrigerator at $-80^{\circ} \mathrm{C}$.

Determination of Peel Color Index. 10 fruits were randomly selected for each treatment, and the color index of the equatorial region of fruits was measured by CR-10 color. And get the index: L* $a^{*}, b^{*}, C^{*}, h^{o} . L^{*}$ is gloss. The greater the $L^{*}$, the higher the gloss of the peel, the lower the contrary. $a^{*}, b^{*}$ are color components, that range $[-60,+60] .+a^{*}$ is red, $-a^{*}$ is green, $+b^{*}$ is yellow, and $-b^{*}$ is blue. The greater the absolute value, the darker the color. $\mathrm{C}^{*}$ is color saturation and $\mathrm{h}^{\mathrm{o}}$ is hue angle. And then calculate the Color Index of Grape (CIRG). $C I R G=\left(180-h^{\circ}\right) /\left(L^{*}+C^{*}\right)$. The fruit color index method was used to evaluate the appearance of fruit color. CIRG $<2$ is yellow-green, $2<\mathrm{CIRG}<4$ is pink, $4<\mathrm{CIRG}<5$ is red, $5<\mathrm{CIRG}<6$ is dark red, and CIRG $>6$ is blue-black ${ }^{[6]}$.

Determination of Total Chlorophyll and Anthocyanin in Pericarp. Anthocyanins, chlorophyll, and carotene are measured by spectrophotometry. Method reference to Qinge Xiong ${ }^{[7]}$.

\section{Results and Discussion}

Effects of Different Concentrations of SUNRED on Anthocyanin Content in 'Red Globe' Grape Fruit. As can be seen from FIG. 1, after the treatment, the anthocyanin content gradually increased with the accumulation of anthocyanins. Before July 25, there was no significant difference between treatments. After July 25 (second spraying), the anthocyanin content of all treatments was higher than control group, and the anthocyanin content at the 1000 times treatment was significantly higher than control group. On August 19th, the anthocyanin content in various treatments increased rapidly, and the anthocyanin content was the highest at 1000 times treatment, which was significantly different from control group, which was $1785 \mathrm{mg} / \mathrm{g}$. This shows that $S U N R E D$ promotes the coloration of grape peel, and SUNRED 1000 times was the best effect.

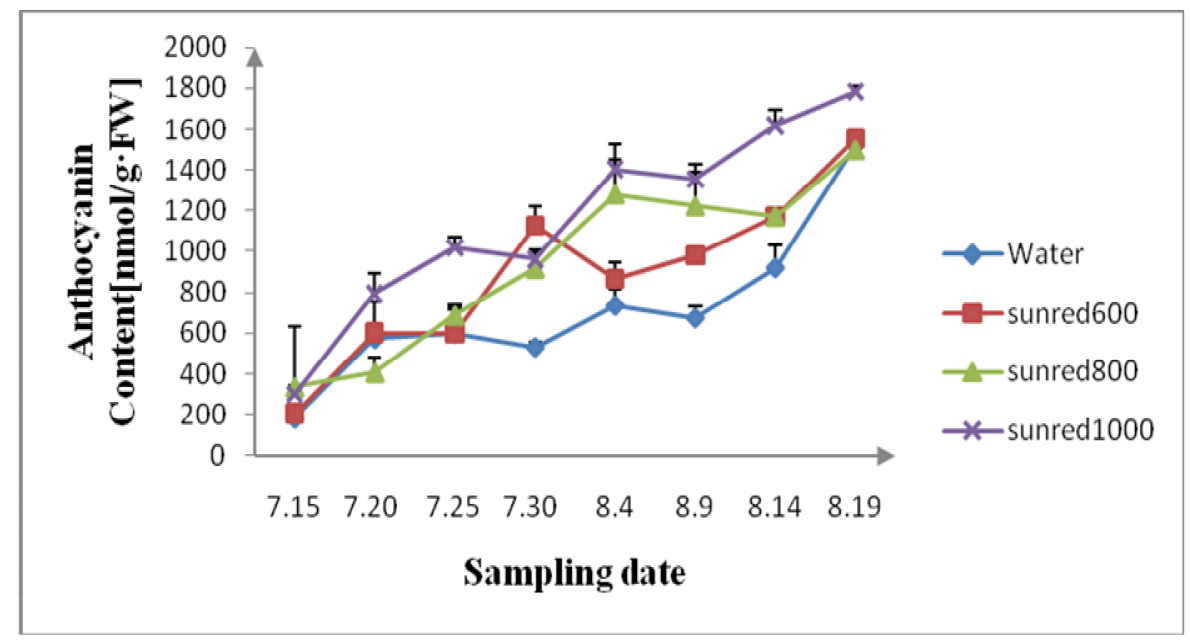

Figure 1. Effects of different concentrations SUNRED on the anthocyanin of 'Red Globe' grape 
Effects of Different Concentrations of SUNRED on the Total Chlorophyll Content of 'Red Globe'Grape Peel. The total chlorophyll of peel can affect the color of peel to a certain extent.As shown in Figure 2, the total chlorophyll content of the peel was gradually reduced with the maturity of the fruits in all treat. On July 20th and August 4th, due to rainfall, the grapes turned green and the total chlorophyll content of the peel increased rapidly. On the fruit maturity of August 19, the total chlorophyll content of the peel was the lowest, and the 1000 times treatment was the lowest, which was significantly different from the control group. This shows that SUNRED can reduce the total chlorophyll content of peel in the fruit maturity, SUNRED1000 times the best effect.

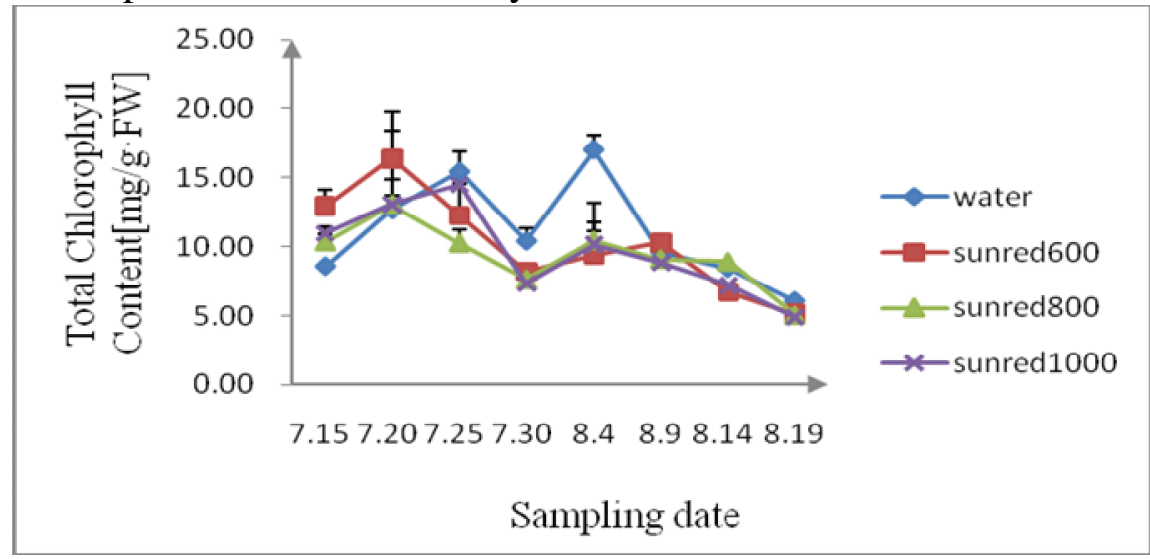

Figure 2. Effects of different concentrations SUNRED on the chlorophyll of 'Red Globe' grape Effects of Different Concentrations of SUNRED on the Color Indexes of Peel of 'Red Globe' Grape. Different concentrations of SUNRED has an effect on the color of peel. As shown in Fig. 3, with the ripening of grape fruits, the $\mathrm{L}^{*}$ of all treated peels gradually decreased. The SUNRED 1000 times maturation period was the lowest, which was 25.11 , which was significantly different from the other treats. At the fruit maturity, The SUNRED 1000 times was the lowest, which was 25.11, which was significantly different from the other treats. This indicates that SUNRED 1000 times liquid can reduce the gloss of the peel. At the same time, with the turning of the fruits, the a* of the treated peels gradually increased and the $\mathrm{b}^{*}$ gradually decreased. At the fruit maturity, $\mathrm{a}^{*}$ was water $>S U N R E D$ 1000 times $>$ SUNRED 600 times $>$ SUNRED 800 times, b* was water $>S U N R E D 600$ times $>$ SUNRED 800 times $>$ SUNRED 1000 times, C was SUNRED 1000 times $>$ SUNRED 600 times $>$ water $>S U N R E D 800$ times. This indicates that peel color of 800 times was the lightest, peel color of water was dark but uneven and 1000 times was the most uniform coloring. CIRG is Color Index of Grape. The figure shows that in the fruit maturity, water, SUNRED 600 times, SUNRED 800 times, SUNRED 1000 times were respectively 4.9, 4.35, 4.81, 5.22, and the difference was significant. the fruit surface color were red, red, red and dark red. In summary, SUNRED 1000 times treat could significantly promote the peel coloring, and make the skin color mature and the color evenly.

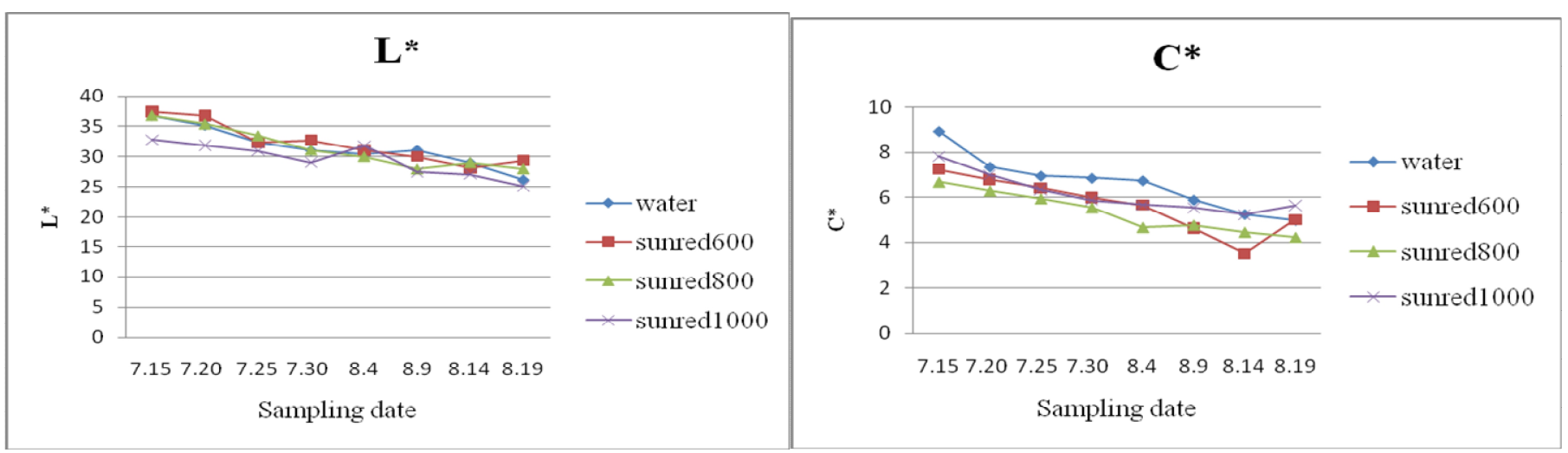



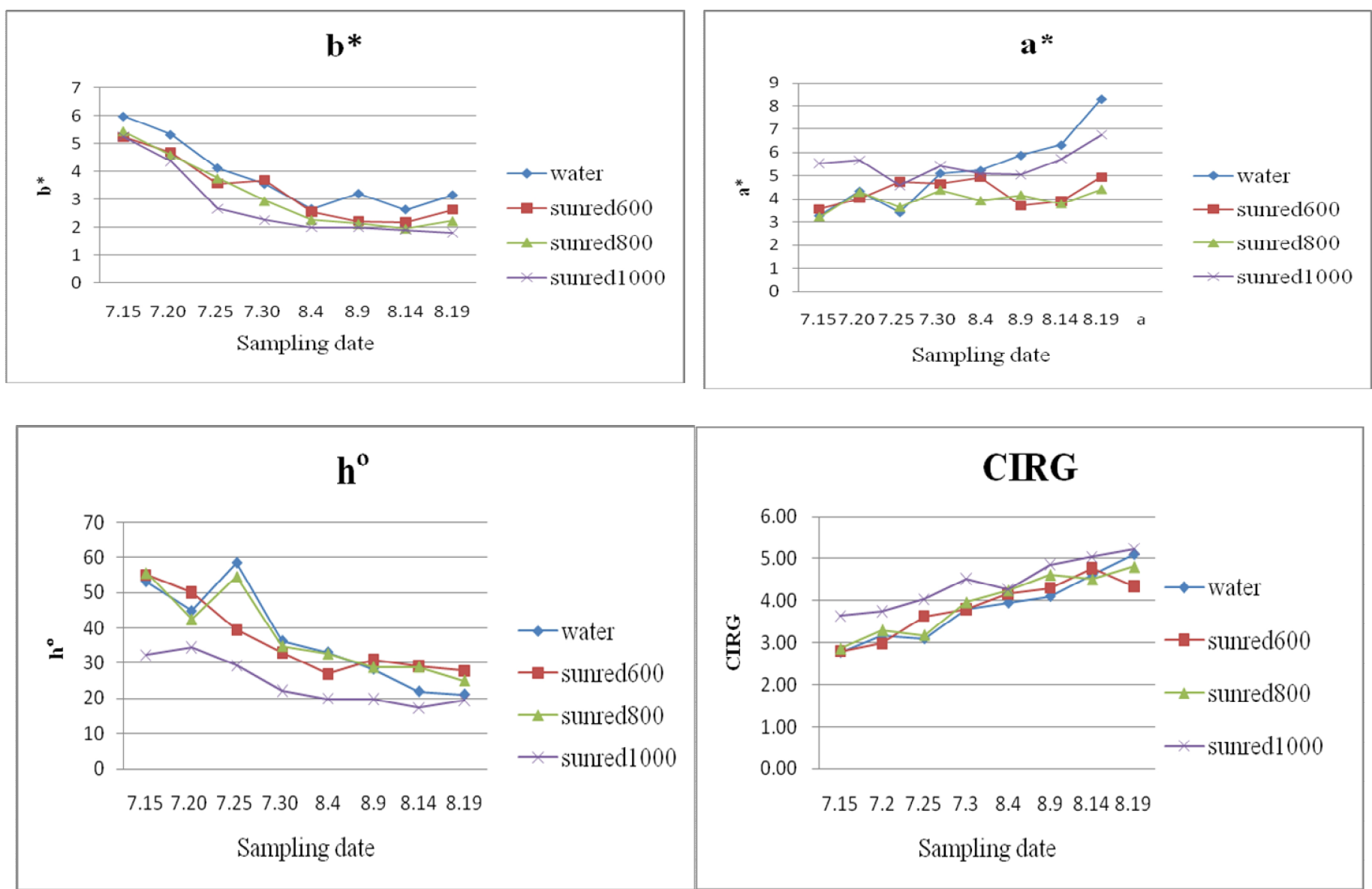

Figure 3. Effects of different concentrations SUNRED on the color index of the peel of'Red

Globe'grape

\section{Conclusions}

Grapes in the southern regions of China are generally difficult to color, especially in grapes grown under high-temperature, rainy southern rain-preventing cultivation ${ }^{[8]}$.Plant growth regulators have been widely used in the production of grapes for a long period of time and have positive effects on promoting fruit coloration, increasing yield, and improving fruit quality ${ }^{[9]}$. However, there are few studies on the effects of seaweed fertilizers on the coloration of grape fruits. In the current situation of organic agricultural development and environmental protection, exploiting seaweed fertilizer as an alternative to chemical fertilizers and plant hormones becoming more and more important ${ }^{[10]}$. As a new seaweed fertilizer, $S U N R E D$ can not only improve the economic value of products, but also play a role in the sustainable development and environmental protection of organic agriculture.

Spraying SUNRED can significantly promote the coloration of 'Red Globe' grapes. After comprehensive analysis, it was believed that SUNRED 1000 times treatment significantly promoted the accumulation of anthocyanins in the pericarp, making the pericarp dark red and evenly colored. In recent years, there are more and more researches on plant growth regulators have been conducted, but still few studies on the application of seaweed fertilizers. In the future, in-depth quality control research will be carried out for different grape varieties, different climatic ecological regions and different grape development periods. To lay a solid foundation for comprehensive improvement of the quality and coloring of Chengdu Plain grapes ${ }^{[11]}$.

\section{Acknowledgements}

This work was financially supported by Sichuan Science and Technology Infrastructure Project "Research on the Digital Descriptive Standard of Grape Resources and the Construction of Sharing Platform” (Item No. 18PTDJ0140) and Sichuan Provincial Agricultural Science and Technology 
Achievements Transformation Fund Project "Xiahei's Promotion and Standardization Demonstration Base for New Grape Varieties"

\section{References}

[1]Junpeng Ren, XiaohongLi, Ruiqi Dong: Effects of girdling and abscisic acid treatment on fruit coloring and related gene expression in 'xiahei' grapes. Journal of Fruit Science. CSCD. Forum Vol. 30(2013), p968-974

[2] Yuanbin Cheng, Hao Fu, Tongxia Wang: Grape shelter cultivation and pest control technology. Northern Fruits. Forum Vol. 3(2014), p24-25

[3] Weiquan Li, Xiangcheng Wang, Huanjin Yang: Grape rain cultivation techniques. The Journal of Hebei Forestry Science and Technology. Forum Vol. 2(2013), p79-80

[4] Peijing Liu: Development of a New Type of Algae Biofertilizer Organic Liquid Fertilizer and Its Fertilizer Efficiency (thesis of master digree: Chinese Academy of Agricultural Sciences, Beijing China 2012)

[5] Gang Liu, Guiming Hou, Jun Liu, Xiaoxia Wen, Hao Qin, Aifeng Zhou, Hexian Zhao: Effect of Seaweed Fertilizer on Yield and Quality of Muskmelon in Greenhouse. Shandong Agricultural Sciences. Forum Vol. 46(2014), p81-82

[6] Xiangyun Meng, Zhicui Wang, Yuge Wang: The effect of ground shading on the coloring of the 'Red Earth' grape in Xinjiang. Journal of Fruit Science. CSCD. Forum Vol. 31(2014), p60-65

[7] Qinge Xiong: Plant physiology experiment tutorial ( $1^{\text {st }}$ edition, Sichuan Science and Technology Press, Chengdu, china 2003)

[8] Yun Cheng, Xinxin Wu, Baijia Lin: The effect of exogenous abscisic acid on the coloration and quality of Wei Ke grape. Jiangsu Agricultural Sciences. Forum Vol. 42(2014), p163-166

[9] Haibo Wang, Xiaoyu Wang, Zhiqiang Hao: Research progress in grape cultivation in China. Chinese fruit trees. CSCD. Forum Vol. 1(2013), p62-64,67

[10] Cheng Ge, Wei Wu: China's microbial fertilizer production, application and problems. Chinese Agricultural Science Bulletin. CSCD. Forum Vol. 10(1994), p24-28

[11] Liangqiang Zhou, Yongliang Cui, Zuqiang Cheng: Effects of different treatments on the coloration and fruit quality of Kyoho grapes. Resource Development and Market. CSSCI. Forum Vol. 29(2013), p666-668 